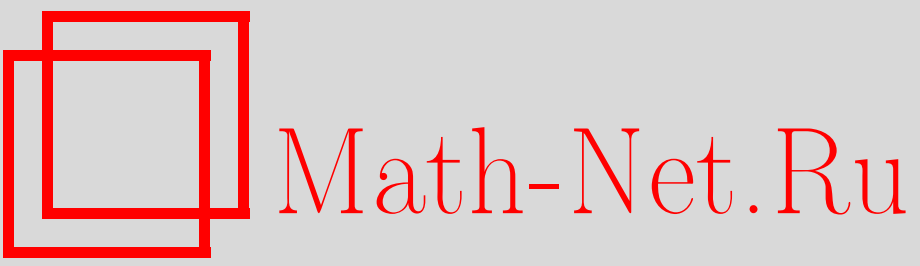

П. В. Денисов, Об асимптотике решения задачи Дирихле для уравнения Лапласа в полупространстве, Итоги науки и техн. Сер. Соврем. мат. и ее прил. Темат. обз., 2020, том 187, 44-49

DOI: https://doi.org/10.36535/0233-6723-2020-187-44-49

Использование Общероссийского математического портала Math-Net.Ru подразумевает, что вы прочитали и согласны с пользовательским соглашением

http://www.mathnet.ru/rus/agreement

Параметры загрузки:

IP : 18.234 .156 .22

26 апреля 2023 г., 12:00:48 


\title{
ОБ АСИМПТОТИКЕ РЕШЕНИЯ ЗАДАЧИ ДИРИХЛЕ ДЛЯ УРАВНЕНИЯ ЛАПЛАСА В ПОЛУПРОСТРАНСТВЕ
}

\author{
(c) 2020 г. П. В. ДЕНИСОВ
}

\begin{abstract}
АннотАция. Работа посвящена изучению асимптотического поведения при больших значениях переменной $y$ решения задачи Дирихле для уравнения Лапласа в полупространстве $E_{+}^{N+1} \equiv$ $\left\{x, y: x \in E^{N}, y>0\right\}$.
\end{abstract}

Ключевые слова: задача Дирихле, полупространство, асимптотическое поведение решений.

\section{ASYMPTOTICS OF SOLUTIONS OF THE DIRICHLET PROBLEM FOR THE LAPLACE EQUATION IN THE HALF-SPACE}

\author{
(c) 2020 P. V. DENISOV
}

\begin{abstract}
This paper is devoted to the study of the asymptotic behavior of the solution of the Dirichlet problem for the Laplace equation in the half-space $E_{+}^{N+1} \equiv\left\{x, y: x \in E^{N}, y>0\right\}$ for large values of the variable $y$.
\end{abstract}

Keywords and phrases: Dirichlet problem, half-space, asymptotic behavior of solutions.

AMS Subject Classification: 35J05, 35C20

В полупространстве $\bar{E}_{+}^{N+1} \equiv\left\{x, y: x \in E^{N}, y \geqslant 0\right\}$ рассмотрим задачу Дирихле для уравнения Лапласа:

$$
\begin{array}{ll}
\frac{\partial^{2} u(x, y)}{\partial y^{2}}+\Delta u(x, y)=0 & (x, y) \in E_{+}^{N+1}, \\
u(x, 0)=u_{0}(x), & x \in E^{N},
\end{array}
$$

где

$$
\Delta=\sum_{k=1}^{N} \frac{\partial^{2}}{\partial x_{k}^{2}} .
$$

Предполагается, что граничная функция $u_{0}(x)$ является непрерывной и ограниченной в $E^{N}$ :

$$
u_{0}(x) \in C\left(E^{N}\right), \quad\left|u_{0}(x)\right| \leqslant C .
$$

Под решением задачи Дирихле (1), (2) будем понимать ограниченное классическое решение задачи $(1),(2)$, т.е. функция $u(x, y)$ дважды непрерывно дифференцируема по $x$ и $y$ в $E_{+}^{N+1}$ и удовлетворяет в классическом смысле уравнению (1) и граничному условию (2).

Автор выражает глубокую признательность проф. М. В. Шамолину за внимание и ценные советы. 
При сделанных предположениях ограниченное решение задачи Дирихле (1), (2) существует, единственно и представимо в виде интеграла Пуассона (см. $[2,4])$ :

$$
u(x, y)=\frac{\Gamma((N+1) / 2) y}{\pi^{(N+1) / 2}} \int_{E^{N}} \frac{u_{0}(z) d z}{\left[y^{2}+|x-z|^{2}\right]^{(N+1) / 2}} .
$$

Символом $S_{R}^{\alpha} u_{0}(x)$ обозначим шаровые средние Рисса порядка $\alpha \geqslant 0$ от граничной функции $(2)$ (cм. $[1,5])$ :

$$
\begin{aligned}
S_{R}^{\alpha} u_{0}(x)=\frac{2}{B(\alpha+1, N / 2) w_{N} R^{N}} \int_{r \leqslant R}\left(1-\frac{r^{2}}{R^{2}}\right)^{\alpha} u_{0}(y) d y= & \\
& =\frac{2}{B(\alpha+1, N / 2) R^{N}} \int_{0}^{R} \rho^{N-1} M\left(x, \rho ; u_{0}\right)\left(1-\frac{\rho^{2}}{R^{2}}\right)^{\alpha} d \rho,
\end{aligned}
$$

где

$$
r=|x-y|=\left[\sum_{k=1}^{N}\left(x_{k}-y_{k}\right)^{2}\right]^{1 / 2}, \quad w_{N}=\frac{2 \pi^{N / 2}}{\Gamma(N / 2)}
$$

и $M\left(x, \rho ; u_{0}\right)$ - сферическое среднее функции $u_{0}(x)$ по поверхности сферы с центром в точке $x$ радиуса $\rho$ :

$$
M\left(x, \rho ; u_{0}\right)=\frac{1}{w_{N} \rho^{N-1}} \int_{|x-y|=\rho} u_{0}(y) d S_{y} .
$$

Изучим вопрос о достаточных условиях существования при некотором $\nu>0$ асимптотики решения задачи Дирихле (1), (2) при $y \rightarrow+\infty$, т.е. вопрос о существовании предела

$$
\lim _{y \rightarrow \infty} y^{\nu} u(x, y)=A(x),
$$

и покажем, что эти условия выражаются в терминах существования шаровых средних Рисса порядка $\alpha \geqslant 0$, согласованной степенной асимптотики по $R$, при $R \rightarrow+\infty$.

Вначале выясним важные свойства предельной функции $A(x)$ в равенстве $(7)$.

Теорема 1. Если предел (7) существует равномерно относительно $x$ на каждом компакте $K$ в $E^{N}$, то функция $A(x)$ является гармонической функцией в $E^{N}$.

Следствие 1. Если и $u_{0}(x)$ - ограниченнал, непрерывная функиия в $E^{N}$, в задаче (1),(2) и предел (7) существует равномерно по $x$ на каждом компакте $K$ в $E^{N}$, то $A(x)=A$-постоянная в $E^{N}$.

Теорема 2. Если граничная функция $u_{0}(x)$ непрерывна и ограничена в $E^{N}$ и шаровые средние Рисса (5) порядка $\alpha$ от $u_{0}(x)$ имеют при некотором $\nu>0$, удовлетворяюоем неравенствам

$$
0<\nu<N+2 \alpha+2,
$$

асимптотику

где

$$
S_{R}^{\alpha} u_{0}(x)=\frac{A}{R^{\nu}}+\frac{Q(x, R)}{R^{\nu}}
$$

$$
\lim _{R \rightarrow+\infty} Q(x, R)=0
$$

равномерно на каждом компакте $K \in E^{N}$ или равномерно по $x$ во всем $E^{N}$, то решение задачи Дирихле (1), (2) имеет при $y \rightarrow+\infty$ асимптотику

где

$$
u(x, y)=\frac{A \cdot C_{1}}{y^{\nu}}+\frac{Q_{1}(x, y)}{y^{\nu}}
$$

$$
\lim _{y \rightarrow+\infty} Q_{1}(x, y)=0
$$


существует равномерно по $x$ на каждом компакте $K \in E^{N}$ или равномерно по $x$ во всем $E^{N}$,

$$
C_{1}=B\left(\frac{N+2 \alpha+2-\nu}{2}, \frac{1+\nu}{2}\right) / B\left(\frac{N+2 \alpha+2}{2}, \frac{1}{2}\right) .
$$

Доказательство теоремъ 1. Пусть шар $\{|x-z| \leqslant R\}$ целиком принадлежит компакту $K$, для которого существует равномерный по $x$ предел (7). Введем обозначение

$$
V(x, y)=y^{\nu} u(x, y) .
$$

Для функции (14), очевидно принадлежащей классу $C^{2}(K)$, имеет место формула

$$
V(x, y)=\frac{1}{w_{N} R^{N-1}} \int_{r=R} V(z, y) d S_{z}+\frac{1}{(N-2)} \int_{r \leqslant R}\left(r^{2-N}-R^{2-N}\right) \Delta V(z, y) d z,
$$

где $r=|x-z|$ (см. [2]). Из этой формулы, уравнения (1) и из существования равномерного на $K$ предела (7) следует, что существует равномерный по $x \in K$ предел

$$
\lim _{y \rightarrow+\infty} \int_{r \leqslant R}\left(r^{2-N}-R^{2-N}\right)\left[y^{\nu} u_{y y}(z, y)\right] d z=B(x) .
$$

Докажем, что предел (16) равен нулю хотя бы для одной последовательности $y_{n} \rightarrow+\infty$. Используя формулу (15), существование равномерных пределов (7), (16), а также интегрирование по частям (см. [3]), получим

$$
\begin{aligned}
\lim _{n \rightarrow+\infty} \int_{n}^{\tau+1} d \tau \int_{\tau}^{n+1}\left[\int_{r \leqslant R}\left(r^{2-N}-R^{2-N}\right) y^{\nu} u_{y y}(z, y) d z\right] d y & \\
& =\lim _{n \rightarrow+\infty} \int_{r \leqslant R}\left(r^{2-N}-R^{2-N}\right)\left[\int_{n}^{n+1} d \tau \int_{\tau}^{\tau+1} y^{\nu} u_{y y}(z, y) d y\right] d z
\end{aligned}
$$

Докажем, что

$$
\lim _{n \rightarrow+\infty} \int_{n}^{n+1} d \tau \int_{\tau}^{\tau+1} y^{\nu} u_{y y}(z, y) d y=0, \quad|x-z| \leqslant R .
$$

Интегрируя по частям, получим

$$
\begin{aligned}
& K=\int_{n}^{n+1} d \tau \int_{\tau}^{\tau+1} y^{\nu} u_{y y}(z, y) d y= \\
&=\left[(n+2)^{n} u(z, n+2)-(n+1)^{n} u(z, n+1)\right]-\left[(n+1)^{n} u(z, n+1)-n^{n} u(z, n)\right]- \\
&\left.\quad-\nu \int_{n}^{n+1}(\tau+1)^{\nu-1} u(z, \tau+1)-\nu^{\nu-1} u(z, \tau)\right) d \tau+ \\
& \quad+\nu(\nu-1) \int_{n}^{n+1} d \tau \int_{\tau}^{\tau+1} y^{\nu-2} u(z, y) d y=K_{1}+K_{2}+K_{3}+K_{4} .
\end{aligned}
$$

Из существования равномерного предела (7) следует, что

$$
\lim _{n \rightarrow \infty} K_{1}=\lim _{n \rightarrow+\infty} K_{2}=0 .
$$


Применяя в $K_{3}$ и $K_{1}$ в $(18)$ первую формулу среднего значения (см. [3, т. 1]) и переходя к пределу при $n \rightarrow \infty$, получим, что существует предел

$$
\begin{aligned}
\lim K_{3}+\lim K_{4}=\lim _{n \rightarrow \infty} \nu\left(\tau_{*}+1\right)^{\nu} u\left(z, \tau_{*}+1\right)-\tau_{*}^{\nu} u\left(z, \tau_{*}\right) & {\left[\ln \frac{n+1}{n+1}-\ln \frac{n+1}{n}\right]+} \\
& +\lim _{n \rightarrow \infty} \nu(\nu-1) \tau_{* *}^{\nu} u\left(z, \tau_{* *}\right) \int_{n}^{n+1} \int_{\tau}^{\tau+1} \frac{d y}{y^{2}}=0
\end{aligned}
$$

т.е. доказано, что существует предел при $y_{n} \rightarrow \infty$ в формуле $(16)$, и предел $B(x)$ равен нулю.

Таким образом доказано, что предельная функция в (7) удовлетворяет формуле среднего арифметического

$$
A(x)=\frac{1}{w_{N} R^{N-1}} \int_{|x-z|=R} A(z) d S_{z} .
$$

Тогда из $[2,4]$ следует, что $A(x)$ является гармонической в $E^{N}$. Теорема 1 доказана.

Доказательство следствия 1. Так как $u_{0}(x)$ по условию непрерывна и ограничена в $E^{N}$, решение (4) задачи Дирихле также является ограниченным в $E^{N+1}$. Тогда из теоремы Лиувилля (см. [2]) и теоремы 1 получим, что $A(x)=A$ - постоянная в $E^{N}$.

Лемма 1. Если граничная функция $u_{0}(x)$ непрерывна и ограничена в $E^{N}$, то для решения задачи Дирихле (1), (2) справедлива формула

$$
u(x, y)=C y \int_{0}^{+\infty} \rho^{N+2 \alpha+1} S_{\rho}^{\alpha} u_{0}(x){\frac{d \rho}{\left[\rho^{2}+y^{2}\right]}}^{(N+2 \alpha+3) / 2},
$$

где

$$
C=2 / B\left(\frac{N+2 \alpha+2}{2}, \frac{1}{2}\right)
$$

и $S_{\rho}^{\alpha} u_{0}(x)$ - шаровые средние Рисса порядка $\alpha$ от $u_{0}(x)$, определенные в (5).

Доказательство. Переходя к сферическим $N$-мерным координатам под знаком интеграла Пуассона, получим

$$
u(x, y)=\frac{2 y}{B(N / 2,1 / 2)} \int_{0}^{+\infty} \frac{\rho^{N-1} M\left(x, \rho ; u_{0}\right) d \rho}{\left[\rho^{2}+y^{2}\right]^{(N+1) / 2}},
$$

где $M\left(x, \rho ; u_{0}\right)$ - сферическое среднее (6) граничной функции $u_{0}(x)$. Совершая под знаком последнего интеграла замену переменной $\rho=y z$, а затем выполняя интегрирование по частям, получим

$$
\begin{aligned}
u(x, y)=\frac{2 y}{B(N / 2,1 / 2)} \int_{0}^{+\infty} \frac{d}{d z}\left[\int_{0}^{z} \sigma^{N-1} M\left(x, y \sigma ; u_{0}\right)\right]\left(1+z^{2}\right)^{-(N+1) / 2} d z= \\
=\left.\frac{2}{B(N / 2,1 / 2)} \lim _{z \rightarrow+\infty}\left(1+z^{2}\right)^{-(N+1) / 2} \cdot \int_{0}^{z} \sigma^{N-1} M\left(x, y \sigma ; u_{0}\right) d \sigma\right|_{z=0} ^{2}+ \\
\quad+\frac{2(N+1)}{B(N / 2,1 / 2)} \int_{0}^{+\infty} z\left(1+z^{2}\right)^{-(N+3) / 2}\left[\int_{0}^{z} \sigma^{N-1} M\left(x, y \sigma ; u_{0}\right) d \sigma\right] d z .
\end{aligned}
$$


Так как $u_{0}(x)$ непрерывна и удовлетворяет условию ограниченности (3), подстановка при $z \rightarrow+\infty$, очевидно, обращается в нуль, поэтому получим

$$
u(x, y)=\frac{2(N+1)}{B(N / 2,1 / 2)} \int_{0}^{+\infty} z\left(1+z^{2}\right)^{-(N+3) / 2}\left[\int_{0}^{z} \sigma^{N-1} M\left(x, y \sigma, u_{0}\right) d \sigma\right] d z .
$$

Проинтегрируем по частям правую часть этого неравенства, при этом аналогично получим

$$
\begin{gathered}
u(x, y)=\frac{2(N+1)}{B(N / 2,1 / 2)} \int_{0}^{\infty}\left(1+z^{2}\right)^{-(N+3) / 2} \frac{d}{d z}\left[\int_{0}^{z} \sigma_{1} d \sigma_{1} \int_{0}^{\sigma_{1}} \sigma^{N-1} M\left(x, y \sigma, u_{0}\right) d \sigma\right] d z= \\
=\frac{2(N+1)(N+3)}{B(N / 2,1 / 2)} \int_{0}^{+\infty}\left(1+z^{2}\right)^{-(N+5) / 2} z\left[\int_{0}^{z} \sigma_{1} d \sigma_{1} \int_{0}^{\sigma_{1}} \sigma^{N-1} M\left(x, y \sigma, u_{0}\right) d \sigma\right] d z .
\end{gathered}
$$

При этом мы учли, что в силу непрерывности и ограниченности функции $u_{0}(x)$ и убывания функции $\left(1+z^{2}\right)^{-(N+3) / 2}$ при $z \rightarrow+\infty$, подстановки при $z \rightarrow+\infty$ обратились в нуль. Продолжая $n$ раз аналогичный процесс интегрирования по частям и учитывая на каждом шаге факт обращения подстановок в нуль, получим формулу:

$$
\begin{aligned}
u(x, y)=\frac{2(N+1)(N+3) \ldots(N+2 n+1)}{B(N / 2,1 / 2)} \int_{0}^{\infty} \frac{z}{\left(1+z^{2}\right)^{(N+2 n+3) / 2}} \times \\
\times\left(\int_{0}^{z} \sigma_{n+1} d \sigma_{n+1} \int_{0}^{\sigma_{n+1}} \sigma_{n} d \sigma_{n} \int_{0}^{\sigma_{n}} \ldots \sigma_{2} d \sigma_{2} \int_{0}^{\sigma_{2}} \sigma_{1}^{N-1} M\left(x, y \sigma_{1} ; u_{0}\right) d \sigma_{1}\right) .
\end{aligned}
$$

Учитывая известную формулу

$$
\int_{0}^{z} \sigma_{n+1} d \sigma_{n+1} \int_{0}^{\sigma_{n+1}} \ldots \sigma_{2} d \sigma_{2} \int_{0}^{\sigma_{2}} \sigma_{1}^{N-1} M\left(x, y \sigma_{1} ; u_{0}\right) d \sigma_{1}=\frac{1}{2^{n} n !} \int_{0}^{z}\left(z^{2}-\sigma^{2}\right)^{n} \sigma^{N-1} M\left(x, \sigma y ; u_{0}\right) d \sigma_{1}
$$

и преобразуя числовой коэффициент при (21) по рекуррентным формулам для $B$ - и Г-функций (см. [3, т. 2]), получим требуемый результат. Лемма 1 доказана.

Доказательство теоремы 2. В силу регулярности шаровых средних Рисса достаточно доказать теорему 2 для случая, когда порядок средних Рисса (9) является натуральным: $\alpha=n \in N$.

Представляя решение Дирихле (1), (2) по формуле (20), при $\alpha=n$ будем иметь

$$
u(x, y)=C_{1} \int_{0}^{+\infty} \frac{\rho^{N+2 n+1}}{(\rho y)^{\nu}}\left[(\rho y)^{\nu} S_{\rho y}^{n} u_{0}(x)\right] \frac{d \rho}{\left(1+\rho^{2}\right)^{(N+2 n+3) / 2}},
$$

где

$$
C_{1}=B\left(\frac{N+2 n-\nu}{2}, \frac{1+\nu}{2}\right) / B\left(\frac{N+2 n+2}{2}, \frac{1}{2}\right) .
$$

Учитывая, что по условию теоремы существует предел (9), заключаем, что для любого $\varepsilon>0$ существует такое $R_{0}(\varepsilon)>0$, что для всех $R>R_{0}$ справедливо равенство

$$
R^{\nu} S_{R}^{n} u_{0}(x)=A+\varepsilon(x, R),
$$

где $|\varepsilon(x, R)|<\varepsilon / 2$ в точке $x$ или равномерно по $x$ во всем $E^{N}$. 
Выбрав $R>R_{0}$, разобьем интеграл (22) на два интеграла:

$$
\begin{gathered}
y^{\nu} u(x, y)=C_{1} \int_{0}^{\delta(y)} \rho^{N+2 n+1-\nu}\left[(y \rho)^{\nu} S_{y \rho}^{n} u_{0}(x)\right]\left(1+\rho^{2}\right)^{(N+2 n+3) / 2} d \rho+ \\
+C_{1} \int_{\delta(y)}^{+\infty} \rho^{N+2 n+1-\nu}\left[(y \rho)^{\nu} S_{y \rho}^{n} u_{0}(x)\right]\left(1+\rho^{2}\right)^{-(N+2 n+3) / 2} d \rho=J_{1}+J_{2},
\end{gathered}
$$

где $\delta(y)=1 / \sqrt{y}$.

Для оценки второго интеграла $J_{2}$ в (24) применим интегральную формулу среднего значения (см. [3, т. 1]):

$$
J_{2}=C_{1} \int_{\delta(y)}^{+\infty} \rho^{N+2 n+1-\nu}\left(1+\rho^{2}\right)^{-(N+2 n+3) / 2} d \rho \cdot\left[\left(y \rho_{*}\right)^{\nu} S_{y \rho_{*}}^{n} u_{0}(x)\right],
$$

где $\rho_{*} \geqslant \delta(y)$. Так как $y \rho_{*} \geqslant y \delta(y)=\sqrt{y} \rightarrow+\infty$ при $y \rightarrow+\infty$, в силу (23) и того факта, что $\delta(y)=1 / \sqrt{y} \rightarrow 0$ при $y \rightarrow+\infty$, получим, что для любого $\varepsilon>0$ существует такое $y_{1}(\varepsilon)$, что при всех $y \geqslant y_{1}$ имеем

$$
\lim _{y \rightarrow+\infty}\left|J_{2}-A \cdot C_{1}\right|=\left|\varepsilon_{1}(x, y)\right|<\frac{\varepsilon}{2} .
$$

Оценим теперь $J_{1}$ в $(24)$. Очевидно, из оценки $(3)$ для $u_{0}(x)$ и равенства $(24)$ следует оценка

$$
\left|\rho^{\nu} y^{\nu} S_{\rho y}^{n} u_{0}(x)\right|<C_{3} \text {. }
$$

Учитывая в $J_{1}$ эту оценку и тот факт, что $\delta(y) \rightarrow 0$ при $y \rightarrow+\infty$, очевидно, получим, что существует предел

$$
\lim _{y \rightarrow+\infty} J_{1}(x, y)=0
$$

в точке $x$ или равномерно по $x \in E^{N}$. Из $(25)$ и (26) вытекает, что теорема 2 доказана.

Теоремы, аналогичные теоремам 1 и 2 настоящей работы, для задачи Коши для уравнения теплопроводности были доказаны в работе [1].

\section{СПИСОК ЛИТЕРАТУРЫ}

1. Денисов В. Н. Об асимптотике при большом времени решения задачи Коши для уравнения теплопроводности // Докл. РАН. - 1995. - 351, № 6. - С. 736-738.

2. Курант Р., Гилъберт Д. Методы математической физики. Т. 2. - М.-Л.: ГТТИ, 1945.

3. Никольский С. М. Курс математического анализа. - М.: Наука, 1983, 1984.

4. Соболев С. Л. Уравнения математической физики. - М., 1966.

5. Харди Г. Расходящиеся ряды. - М., 1951.

Денисов Петр Васильевич

Московский педагогический государственный университет

E-mail: denisov.piter@gmail.com 